Скопје, Македонија

\title{
A NOTE ON COMPATIBLE BINARY RELATIONS ON VECTOR VALUED HYPERSEMIGROUPS
}

\author{
VALENTINA MIOVSKA AND VESNA CELAKOSKA-JORDANOVA
}

\begin{abstract}
In this note we present some properties concerning the connection between vector valued hypersemigroups and various kinds of compatible binary relations defined on them, i.e. $i$-compatible, compatible, strongly $i$-compatible, strongly compatible, regular and strongly $i$-regular binary relations.
\end{abstract}

Binary hyperstructures were introduced by Marty in [8] as a natural extension of classical algebraic structures. Vector valued hyperstructures were introduced in [9] as a generalization of $n$-ary hyperstructures $([5,2])$ and vector valued structures $([10,6,7])$. Besides the concepts of vector valued hypergroupoids, hypersemigrops, weak hypersemigroups, etc., regular and strongly regular binary relations on vector valued hypersemigroups were introduced in [9] as well. Following some recent papers of Davvaz and Loreanu-Fotea $([1,3,4])$, in this short note we introduce the notions of $i$-compatible, strongly $i$-compatible, $i$-regular relations for some $i \in\{0,1, \ldots, n-1\}$, as well as compatible and strongly compatible relations on vector valued hypersemigroups and prove a few properties concerning these notions. For the sake of completeness, we will repeat the definitions of vector valued hypergroupoid and vector valued hypersemigroup from the paper [9].

Let $H$ be a nonempty set and let $n, m$ be positive integers such that $n \geq m$. Denote by $\mathcal{P}^{*}(H)$ the set of all nonempty subsets of $H$ and by $H^{n}$ the $n$th Cartesian product of $H$.

Definition 1. ([9], Def.1.1.) A mapping [ ] : $H^{n} \rightarrow\left(\mathcal{P}^{*}(H)\right)^{m}$ from the $n$th Cartesian product of $H$ to the $m$ th Cartesian product of $\mathcal{P}^{*}(H)$ is called an

2010 Mathematics Subject Classification. 20N20.

Key words and phrases. Vector valued hypergroupoid, vector valued hypersemigroup, strong homomorphism, binary relation, compatible relation. 
(n,m)-hyperoperation on $H$. If it is not necessary to emphasize the integers $n$ and $m$, then we will say that [] is a vector valued hyperoperation instead of $(n, m)$-hyperoperation.

Throughout the paper, the elements of $H^{n}$, i.e. the sequences $\left(x_{1}, \ldots, x_{n}\right)$ will be denoted by $x_{1} x_{2} \ldots x_{n}$ or, shortly, $x_{1}^{n}$. The symbol $x_{i}^{j}$ will denote the sequence $x_{i} x_{i+1} \ldots x_{j}$ of elements of $H$ when $i \leq j$ and the empty symbol when $i>j$.

Definition 2. ([9], Def.1.2.) A sequence of $m n$-ary hyperoperations [ ] $]_{s}$ : $H^{n} \rightarrow \mathcal{P}^{*}(H), s \in\{1,2, \ldots, m\}$, can be associated to [] by putting

$$
\left[a_{1}^{n}\right]_{s}=B_{s} \Leftrightarrow\left[a_{1}^{n}\right]=\left(B_{1}, \ldots, B_{m}\right),
$$

for all $a_{1}, \ldots, a_{n} \in H$. Then, we call [ ] $]_{s}$ the sth component hyperoperation of [ ] and write [ ] $=\left([]_{1}, \ldots,[]_{m}\right)$. Note that there is a unique $(n, m)$ hyperoperation on $H$ whose component hyperoperations are [ $]_{s}$.

An $(n, m)$-hyperoperation [ ] on $H$ is extended to subsets $A_{1}, A_{2}, \ldots, A_{n}$ of $H$ in a natural way, i.e.

$$
\left[A_{1} A_{2} \ldots A_{n}\right]=\left(\left[A_{1} A_{2} \ldots A_{n}\right]_{1},\left[A_{1} A_{2} \ldots A_{n}\right]_{2}, \ldots,\left[A_{1} A_{2} \ldots A_{n}\right]_{m}\right),
$$

where $\left[A_{1} A_{2} \ldots A_{n}\right]_{s}=\cup\left\{\left[a_{1}^{n}\right]_{s} \mid a_{i} \in A_{i}, i=1,2, \ldots, n\right\}$ and $s=1,2, \ldots, m$.

Clearly, $C_{1}^{p} \subseteq B_{1}^{p}$ if and only if $C_{i} \subseteq B_{i}$, for $i=1, \ldots, p$, and, $x_{1}^{p} \in C_{1}^{p}$ if and only if $x_{i} \in C_{i}$ for $i=1, \ldots, p$.

Definition 3. ([9], Def.1.3.) An algebraic structure $\boldsymbol{H}=(H,[])$, where [ ] is an $(n, m)$-ary hyperoperation defined on a nonempty set $H$, is called an $(n, m)$-hypergroupoid or vector valued hypergroupoid. Identifying the set $\{x\}$ with the element $x$, any $(n, m)$-groupoid is an $(n, m)$-hypergroupoid. If []$=\left([]_{1}, \ldots,[]_{m}\right)$, we denote by $\left(H ;[]_{1}, \ldots,[]_{m}\right)$ the component hypergroupoid of $\boldsymbol{H}$ and $\left(H,[]_{j}\right)$ is the $j$ th component $n$-ary hypergroupoid of $\boldsymbol{H}$.

Further on we assume that the positive integers $n$ and $m$ are such that $n>m$, i.e. $n=m+k$, for $k \geq 1$.

Definition 4. ([9], Def.1.4.) An (n,m)-hyperoperation is said to be associative if

$$
\left[x_{1}^{i}\left[x_{i+1}^{i+n}\right] x_{i+n+1}^{n+k}\right]=\left[x_{1}^{j}\left[x_{j+1}^{j+n}\right] x_{j+n+1}^{n+k}\right],
$$

holds for all $x_{1}, \ldots, x_{n+k} \in H$ and for all $i, j \in\{1,2, \ldots, n\}$.

An $(n, m)$-hyperoperation is said to be weakly associative if

$$
\left[x_{1}^{i}\left[x_{i+1}^{i+n}\right] x_{i+n+1}^{n+k}\right]_{s} \cap\left[x_{1}^{j}\left[x_{j+1}^{j+n}\right] x_{j+n+1}^{n+k}\right]_{s} \neq \emptyset,
$$


holds for all $i, j \in\{1,2, \ldots, n\}, x_{1}, \ldots, x_{n+k} \in H$ and every $s \in\{1,2, \ldots, m\}$.

An $(n, m)$-hypergroupoid with an associative operation (weakly associative operation) is called an ( $n, m)$-hypersemigroup (weak $(n, m)$-hypersemigroup).

Examples of $(n, m)$-hypersemigroups and weak $(n, m)$-hypersemigroups are presented in [9].

Definition 5. ([9], Def.1.10.) Let $(H,[])$ and $\left(H^{\prime},[]^{\prime}\right)$ be $(n, m)$-hypergroupoids. A mapping $\varphi: H \rightarrow H^{\prime}$ is:

a) a strong homomorphism if and only if $\varphi\left(\left[a_{1}^{n}\right]_{s}\right)=\left[\varphi\left(a_{1}\right) \ldots \varphi\left(a_{n}\right)\right]_{s}^{\prime}$;

b) an inclusion homomorphism if and only if $\varphi\left(\left[a_{1}^{n}\right]_{s}\right) \subseteq\left[\varphi\left(a_{1}\right) \ldots \varphi\left(a_{n}\right)\right]_{s}^{\prime}$;

c) a weak homomorphism if and only if $\varphi\left(\left[a_{1}^{n}\right]_{s}\right) \cap\left[\varphi\left(a_{1}\right) \ldots \varphi\left(a_{n}\right)\right]_{s}^{\prime} \neq \varnothing$,

for every $s=1,2, \ldots, n$. The mapping $\varphi$ that is a bijection and strong homomorphism is called an isomorphism, and it is called an automorphism if $\varphi$ is defined on the same $(n, m)$-hypergroupoid.

Theorem 1. Let $\boldsymbol{H}, \boldsymbol{H}_{1}, \boldsymbol{H}_{2}$ be (n,m)-hypersemigroups (weak (n,m)-hypersemigroups), $\varphi_{1}: H \rightarrow H_{1}$ be a surjective strong homomorphism and $\varphi_{2}$ : $H \rightarrow H_{2}$ be a strong homomorphism, such that $\operatorname{ker} \varphi_{1} \subseteq \operatorname{ker} \varphi_{2}$. Then there exist a unique strong homomorphism $\theta: H_{1} \rightarrow H_{2}$ such that $\theta \circ \varphi_{1}=\varphi_{2}$.

Proof. Let $a \in H$. Then $\varphi_{1}(a)=a_{1} \in H_{1}$. Let $\theta: H_{1} \rightarrow H_{2}$ be a mapping defined by $\theta\left(a_{1}\right)=\varphi_{2}(a)$. Let $a_{1}=b_{1}$. Since $\varphi$ is a surjective mapping it follows that there is $b \in H$ such that $\varphi_{1}(b)=b_{1}$. Clearly, $\varphi_{1}(a)=\varphi_{1}(b)$, i.e. $(a, b) \in \operatorname{ker} \varphi_{1} \subseteq \operatorname{ker} \varphi_{2}$. Thus, $\varphi_{2}(a)=\varphi_{2}(b)$, i.e. $\theta\left(a_{1}\right)=\theta\left(b_{1}\right)$. Hence, $\theta$ is a well defined mapping and

$$
\left(\theta \circ \varphi_{1}\right)(a)=\theta\left(\varphi_{1}(a)\right)=\theta\left(a_{1}\right)=\varphi_{2}(a) .
$$

The mapping $\theta$ is a strong homomorphism. Namely, for every $s \in$ $\{1,2, \ldots, m\}$

$\theta\left(\left[a_{1}^{n}\right]_{s}\right)=\theta\left(\left[\varphi_{1}\left(a_{1}^{\prime}\right) \ldots \varphi_{1}\left(a_{n}^{\prime}\right)\right]_{s}\right)=\theta\left(\varphi_{1}\left(\left[a_{1}^{\prime} \ldots a_{n}^{\prime}\right]_{s}\right)\right)=\varphi_{2}\left(\left[a_{1}^{\prime} \ldots a_{n}^{\prime}\right]_{s}\right)=$
$=\left[\varphi_{2}\left(a_{1}^{\prime}\right) \ldots \varphi_{2}\left(a_{n}^{\prime}\right)\right]_{s}=\left[\left(\theta \circ \varphi_{1}\right)\left(a_{1}^{\prime}\right) \ldots\left(\theta \circ \varphi_{1}\right)\left(a_{n}^{\prime}\right)\right]_{s}=\left[\theta\left(a_{1}\right) \ldots \theta\left(a_{n}\right)\right]_{s}$.

Suppose that there is a strong homomorphism $\theta_{1}: H_{1} \rightarrow H_{2}$ such that $\theta_{1} \circ \varphi_{1}=\varphi_{2}$. Let $a_{1} \in H_{1}$. Then $\theta_{1}\left(a_{1}\right)=\theta_{1}\left(\varphi_{1}(a)\right)=\left(\theta_{1} \circ \varphi_{1}\right)(a)=$ $\varphi_{2}(a)=\left(\theta \circ \varphi_{1}\right)(a)=\theta\left(\left(\varphi_{1}\right)(a)\right)=\theta\left(a_{1}\right)$, i.e. $\theta$ is a unique strong homomorphism. 
Let $H$ be a nonempty set. Denote by $B(H)$ the set of all binary relations on $H$, by $E(H)$ the set of all equivalence relations on $H$.

Definition 6. Let $(H,[])$ be an $(n, m)$-hypersemigroup. A relation $\rho \in$ $B(H)$ is said to be:

a) $i$-compatible, where $i \in\{0,1, \ldots, n-1\}$, if for any $a, b \in H$ and $s=1, \ldots, m$

$$
\left(a \rho b \wedge x \in\left[x_{1}^{i} a x_{i+2}^{n}\right]_{s}\right) \Rightarrow\left(\exists y \in\left[x_{1}^{i} b x_{i+2}^{n}\right]_{s}\right) x \rho y .
$$

Specially, for $i=0(i=n-1)$ we say that $\rho$ is right (left) compatible.

b) compatible if for every $j=1,2, \ldots, n$ and $s=1, \ldots, m$

$$
\left(a_{j} \rho b_{j} \wedge x \in\left[a_{1}^{n}\right]_{s}\right) \Rightarrow\left(\exists y \in\left[b_{1}^{n}\right]_{s}\right) x \rho y .
$$

c) strongly $i$-compatible if for any $a, b \in H$

$$
a \rho b \Rightarrow x \rho y,
$$

for every $x \in\left[x_{1}^{i} a x_{i+1}^{n}\right]_{s}, y \in\left[x_{i} b x_{i+1}^{n}\right]_{s}$. Specially, for $i=0(i=n-1)$ we say that $\rho$ is strongly right compatible (strongly left compatible).

d) strongly compatible if the following implication holds:

$$
(\forall j=1, \ldots, n) a_{j} \rho b_{j} \Rightarrow x \rho y,
$$

for every $x \in\left[a_{1}^{n}\right]_{s}, y \in\left[b_{1}^{n}\right]_{s}, s=1, \ldots, m$.

If $\rho \in E(H)$ and it is $i$-compatible, compatible, strongly $i$-compatible and strongly compatible $(i \in\{0,1, \ldots, n-1\})$, then $\rho$ is said to be $i$-regular, regular, strongly i-regular, strongly regular, respectively.

Example 1. Let $H=\mathbb{Z}_{4}$ and [ ] : $H^{3} \rightarrow\left(\mathcal{P}^{*}(H)\right)^{2}$ be a $(3,2)$-hyperoperation defined by:

$$
\left[x_{1}^{3}\right]=\left\{\begin{array}{l}
\left(\{2,3\}, x_{3}\right), \text { if } x_{1}=x_{2}=x_{3}=0 \\
\left(\{1,3\}, x_{3}\right), \text { otherwise }
\end{array}\right.
$$

By a direct verification of each case, one can show: $\left[\left[x_{1}^{3}\right] x_{4}\right]=\left(\{1,3\}, x_{4}\right)=$ $\left[x_{1}\left[x_{2}^{4}\right]\right]$, i.e. $(H,[])$ is a $(3,2)$-hypersemigroup.

Let $\rho=\{(1,1),(1,2),(1,3),(3,1)\} \in B(H)$. It can be easily verified that $\rho$ is strongly left compatible (i.e. strongly 2-compatible), since $a \rho b$ implies that $x \rho y$, for every $x \in\left[x_{1}^{2} a\right]_{s}$ and $y \in\left[x_{1}^{2} b\right]_{s}, s=1,2$. For instance, $(1,1) \in \rho$ implies that $x \rho y$, for every $x, y \in\left[x_{1}^{2} 1\right]_{1}=\{1,3\}$ and $x, y \in\left[x_{1}^{2} 1\right]_{2}=1$. This relation is not strongly 0 -compatible or 1 -compatible since, for instance, $\left[\begin{array}{lll}2 & 1 & 0\end{array}\right]_{2}=0,\left[\begin{array}{lll}2 & 2 & 0\end{array}\right]_{2}=0$, but $(0,0) \notin \rho$. 
Example 2. Let $H=\{1,2,3,4\}$ and let [ ] : $H^{4} \rightarrow\left(\mathcal{P}^{*}(H)\right)^{2}$ be a $(4,2)$-hyperoperation defined by $\left[x_{1}^{4}\right]=(\{1,2\},\{3,4\})$. Then $(H,[])$ is a (4,2)-hypersemigroup. Namely:

$$
\begin{aligned}
& {\left[\left[x_{1}^{4}\right] x_{5}^{6}\right]=\left[\{1,2\}\{3,4\} x_{5}^{6}\right]=} \\
= & \left(\left[13 x_{5}^{6}\right]_{1} \cup\left[14 x_{5}^{6}\right]_{1} \cup\left[23 x_{5}^{6}\right]_{1} \cup\left[24 x_{5}^{6}\right]_{1},\left[13 x_{5}^{6}\right]_{2} \cup\left[14 x_{5}^{6}\right]_{2} \cup\left[23 x_{5}^{6}\right]_{2} \cup\left[24 x_{5}^{6}\right]_{2}\right)= \\
= & (\{1,2\},\{3,4\}), \\
& {\left[x_{1}\left[x_{2}^{5}\right] x_{6}\right]=\left[x_{1}\{1,2\}\{3,4\} x_{6}\right]=} \\
= & \left(\left[x_{1} 13 x_{6}\right]_{1} \cup\left[x_{1} 14 x_{6}\right]_{1} \cup\left[x_{1} 23 x_{6}\right]_{1} \cup\left[x_{1} 24 x_{6}\right]_{1},\right. \\
& {\left.\left[x_{1} 13 x_{6}\right]_{2} \cup\left[x_{1} 14 x_{6}\right]_{2} \cup\left[x_{1} 23 x_{6}\right]_{2} \cup\left[x_{1} 24 x_{6}\right]_{2}\right)=(\{1,2\},\{3,4\}), } \\
& {\left[x_{1}^{2}\left[x_{3}^{6}\right]\right]=\left[x_{1}^{2}\{1,2\}\{3,4\}\right]=} \\
= & \left(\left[x_{1}^{2} 13\right]_{1} \cup\left[x_{1}^{2} 14\right]_{1} \cup\left[x_{1}^{2} 23\right]_{1} \cup\left[x_{1}^{2} 24\right]_{1},\left[x_{1}^{2} 13\right]_{2} \cup\left[x_{1}^{2} 14\right]_{2} \cup\left[x_{1}^{2} 23\right]_{2} \cup\left[x_{1}^{2} 24\right]_{2}\right)= \\
= & (\{1,2\},\{3,4\}) .
\end{aligned}
$$

Let $\rho=\{(1,1),(2,2),(3,3),(4,4),(1,2),(2,1),(3,4),(4,3)\}$ be an equivalence relation on $H$ and let $a_{j} \rho b_{j}$, for $j=1, \ldots, 4$. Then $\left[a_{1}^{4}\right]_{1}=\left[b_{1}^{4}\right]_{1}=$ $\{1,2\}$ and $\left[a_{1}^{4}\right]_{2}=\left[b_{1}^{4}\right]_{2}=\{3,4\}$. For every $x \in\left[a_{1}^{4}\right]_{s}$ and $y \in\left[b_{1}^{4}\right]_{s}, s=1,2$, one obtains that $x \rho y$ holds. Thus, $\rho$ is a strongly regular relation.

Proposition 1. Let $(H,[])$ be an $(n, m)$-hypersemigroup. If $\rho \in B(H)$ is reflexive and strongly compatible, then $\rho$ is strongly $i$-compatible for every $i \in\{0, \ldots, n-1\}$.

Proof. Let $a \rho b$ for any elements $a, b \in H$ and $x \in\left[x_{1}^{i} a x_{i+2}^{n}\right]_{s}, y \in\left[x_{1}^{i} b x_{i+2}^{n}\right]_{s}$ for every $s=1, \ldots, m$. Since $\rho$ is reflexive, $x_{j} \rho x_{j}, j \in\{1, \ldots, i, i+2, \ldots, n\}$ and $a \rho b$. The strong compatibility of $\rho$ implies that $a \rho y$.

Proposition 2. Let $(H,[])$ be an $(n, m)$-hypersemigroup and $\rho \in B(H)$ be reflexive and transitive. The relation $\rho$ is strongly compatible if and only if $\rho$ is strongly $i$-compatible for every $i \in\{0, \ldots, n-1\}$.

Proof. The direct statement follows from Prop.1. Conversely, let $\rho$ be a reflexive, transitive and strongly $i$-compatible relation for every $i \in\{0,1, \ldots$, $n-1\}$. Let $a_{j} \rho b_{j}, j=1, \ldots, n, x \in\left[a_{1}^{n}\right]_{s}$ and $y \in\left[b_{1}^{n}\right]_{s}$ for every $s=$ $1, \ldots, m$. Since:

$$
\begin{aligned}
& \left(a_{1} \rho b_{1} \wedge x \in\left[a_{1}^{n}\right]_{s} \wedge x_{1} \in\left[b_{1} a_{2}^{n}\right]_{s}\right) \Rightarrow x \rho x_{1}, \\
& \left(a_{2} \rho b_{2} \wedge x_{1} \in\left[b_{1} a_{2}^{n}\right]_{s} \wedge x_{2} \in\left[b_{1} b_{2} a_{3}^{n}\right]_{s}\right) \Rightarrow x_{1} \rho x_{2}, \\
& \ldots \\
& \left(a_{n} \rho b_{n} \wedge x_{n-1} \in\left[b_{1}^{n-1} a_{n}\right]_{s} \wedge y \in\left[b_{1}^{n}\right]_{s}\right) \Rightarrow x_{n-1} \rho y,
\end{aligned}
$$


and the transitivity of $\rho$, it follows that $x \rho y$.

As a consequence of the previous proposition we obtain the following

Corollary 1. If $\rho \in E(H)$ is a strongly regular relation on $(n, m)$-hypersemigroup $(H,[])$, then $\rho$ is strongly $i$-regular relation for every $i \in\{0, \ldots, n-1\}$.

Proposition 3. Let $(H,[])$ be an $(n, m)$-hypersemigroup. If $\rho, \theta \in B(H)$ are strongly $i$-compatible for some $i \in\{0,1, \ldots, n-1\}$ (strongly compatible), then $\rho \circ \theta$ is strongly $i$-compatible (strongly compatible).

Proof. Let $\rho, \theta \in B(H)$ be strongly $i$-compatible for some $i \in\{0, \ldots, n-1\}$ and $a \rho \circ \theta b, x \in\left[x_{1}^{i} a x_{i+2}^{n}\right]_{s}, y \in\left[x_{1}^{i} b x_{i+2}^{n}\right]_{s}$, for every $s=1, \ldots, m$. Since $a \rho \circ \theta b$, it follows that there exists $c \in H$ such that $a \rho c$ and $c \theta b$. If $z \in\left[x_{1}^{i} c x_{i+1}^{n}\right]_{s}$, then by the strong $i$-compatibility of $\rho$ it follows that $x \rho z$. One can analogously conclude that $z \theta y$ and thus $x \rho \circ \theta y$. Strong compatibility can be shown in a similar way.

Proposition 4. Let $(H,[])$ be an $(n, m)$-hypersemigroup. If the relations $\rho_{j} \in B(H), j \in\{1, \ldots, n\}$, are strongly $i$-compatible for every $i \in$ $\{0, \ldots, n-1\}$, then $\bigcup\left\{\rho_{j} \mid j=1, \ldots, n\right\}$ is strongly $i$-compatible.

Proof. Let $a \bigcup_{j=1}^{n} \rho_{j} b$ and $x \in\left[x_{1}^{i} a x_{i+2}^{n}\right]_{s}, y \in\left[x_{1}^{i} b x_{i+2}^{n}\right]_{s}$, for every $s \in\{1, \ldots, m\}$. Then, there exists $j \in\{1, \ldots, n\}$ such that $a \rho_{j} b$. Since $\rho$ is a strongly $i$-compatible relation it follows that $x \rho_{j} y$ and therefore $x \bigcup_{j=1}^{n} \rho_{j} y$.

Proposition 5. Let $(H,[])$ be an $(n, m)$-hypersemigroup. If the relations $\rho_{j} \in E(H), j \in\{1, \ldots, n\}$, are strongly $i$-regular for every $i \in\{0, \ldots, n-1\}$, then $\bigcap\left\{\rho_{j} \mid j=1, \ldots, n\right\}$ is strongly $i$-regular.

Proof. Let $a \bigcap_{j=1}^{n} \rho_{j} b$ and $x \in\left[x_{1}^{i} a x_{i+2}^{n}\right]_{s}, y \in\left[x_{1}^{i} b x_{i+2}^{n}\right]_{s}$, for every $s \in$ $\{1, \ldots, m\}$. Then, for every $j \in\{1, \ldots, n\}, a \rho_{j} b$. Since $\rho_{j}$ are $i$-regular relations it follows that $x \rho_{j} y$, for every $j$. Therefore, $x \bigcap_{j=1}^{n} \rho_{j} y$.

Theorem 2. Let $\boldsymbol{H}$ and $\boldsymbol{K}$ be two (n,m)-hypersemigroups and $\varphi: H \rightarrow K$ be a strong homomorphism. Then $\rho=\left\{(a, b) \in H^{2} \mid \varphi(a)=\varphi(b)\right\}$ is a regular relation.

Proof. It is obvious that $\rho$ is an equivalence relation on $H$. Let $a_{j} \rho b_{j}$ for every $j \in\{1,2, \ldots, n\}$ and let $x \in\left[a_{1}^{n}\right]_{s}$ for $s \in\{1,2, \ldots, m\}$. Then $\varphi\left(a_{j}\right)=\varphi\left(b_{j}\right)$. Since $x \in\left[a_{1}^{n}\right]_{s}$ it follows that $\varphi(x) \in \varphi\left(\left[a_{1}^{n}\right]_{s}\right)$ and, since $\varphi$ is a strong homomorphism, we obtain that $\varphi(x)=\left[\varphi\left(a_{1}\right) \ldots \varphi\left(a_{n}\right)\right]_{s}=$ 
$\left[\varphi\left(b_{1}\right) \ldots \varphi\left(b_{n}\right)\right]_{s}=\varphi\left(\left[b_{1}^{n}\right]_{s}\right)$. Hence, there exists $y \in\left[b_{1}^{n}\right]$ such that $\varphi(x)=$ $\varphi(y)$, i.e. $x \rho y$. Therefore, $\rho$ is a regular relation.

\section{REFERENCES}

[1] B. Davvaz, Some Results on Congruences on Semihypergroups, Bull. Malaysian Math. Sc. Soc. (Second Seies) 23 (2000) 53-58.

[2] B. Davvaz, W. A. Dudek, T. Vougiouklis, A Generalization of n-ary Algebraic Systems, Communications in Algebra, 37 (2009) 1248-1263.

[3] B. Davvaz, V. Loreanu-Fotea, Binary Relations on Ternary Semihypergroups, Communications in Algebra, 38:10 (2010) 3621-3636.

[4] B. Davvaz, V. Loreanu-Fotea, Ternary Hypergroups (A Survey), Int. Journal of Algebraic Hyperstructures and its Applications, Vol. 1, No.1, (2014), 29-44.

[5] B. Davvaz, T. Vougiouklis, N-ary Hypergroups, Iranian Journal of Science \& Technology, Transaction A, 30 (2006) 165-174.

[6] Ǵ. Čupona, Vector valued semigroups, Semigroup Forum, 26 (1983), 65-74.

[7] Ǵ. Čupona, N. Celakoski, S. Markovski, D. Dimovski, Vector Valued Groupoids, Semigroups and Groups, Vector Valued Groupoids, Semigroups and Groups, Maced. Acad. of Sci. and Arts, 1988, 1-78.

[8] F. Marty, Sur une Generalization de la Notion de Groupe, 8th Congress Math., Scandenaves, Stocholm (1934) 45-49.

[9] V. Miovska, V. Celakoska-Jordanova, B. Davvaz, Vector Valued Hyperstructures, Kragujevac Journal of Mathematics (to appear).

[10] B. Trpenovski, B., Ǵ. Čupona, $[m, n]$-groupoids, Bull. Soc. Math. Phys. Macédoine, 21 (1970), 19-29 (in Macedonian).

Ss. Ciril and Methodius University,

Faculty of Natural Sciences and Mathematics,

Arhidemova 3, 1000 Skopje, R. Macedonia

E-mail address: miovska@pmf.ukim.mk

E-mail address: vesnacj@pmf.ukim.mk, celakoska@gmail.com 